\title{
Whole-body diffusion-weighted imaging for staging malignant lymphoma in children
}

\author{
Thomas C. Kwee • Taro Takahara • \\ Malou A. Vermoolen • Marc B. Bierings • \\ Willem P. Mali • Rutger A. J. Nievelstein
}

Received: 6 August 2009 /Revised: 25 November 2009 /Accepted: 20 December 2009 /Published online: 30 July 2010

(C) The Author(s) 2010. This article is published with open access at Springerlink.com

\begin{abstract}
CT}$ is currently the mainstay in staging malignant lymphoma in children, but the risk of second neoplasms due to ionizing radiation associated with $\mathrm{CT}$ is not negligible. Whole-body MRI techniques and whole-body diffusionweighted imaging (DWI) in particular, may be a good radiation-free alternative to $\mathrm{CT}$. DWI is characterized by high sensitivity for the detection of lesions and allows quantitative assessment of diffusion that may aid in the evaluation of malignant lymphomas. This article will review whole-body MRI techniques for staging malignant lymphoma with emphasis on whole-body DWI. Furthermore, future considerations and challenges in whole-body DWI will be discussed.
\end{abstract}

Keywords Whole-body MRI · Diffusion-weighted imaging $\cdot$ Malignant lymphoma $\cdot$ Children $\cdot$ MRI

\section{Introduction}

Cancer is the second most common cause of death among children aged $0-14$ years, surpassed only by accidents [1]. Of all childhood cancers, the malignant lymphomas [Hodgkin disease (HD) and non-Hodgkin lymphoma (NHL)] rank third in incidence, with an age-standardized incidence rate of 15.5 per million [2]. Furthermore, in

T. C. Kwee $(\bowtie) \cdot$ T. Takahara • M. A. Vermoolen • W. P. Mali R. A. J. Nievelstein

Department of Radiology, University Medical Center Utrecht, Heidelberglaan 100,

3584 CX Utrecht, The Netherlands

e-mail: thomaskwee@gmail.com

\section{B. Bierings}

Department of Pediatric Hematology,

University Medical Center Utrecht,

Utrecht, The Netherlands adolescents (aged 15-19 years) the malignant lymphomas are the leading cause of cancer, with an age-standardized incidence of 47.4 per million [2]. Once a malignant lymphoma has been diagnosed histologically, extent of disease has to be assessed (i.e. staging), because this determines treatment planning and prognosis, and knowing all sites of involvement allows monitoring the effect of therapy $[3,4]$. Malignant lymphomas are staged using the Ann Arbor staging system, except for childhood NHLs that are staged using the Murphy staging system [3-6]. Initial staging (at diagnosis) and restaging (after onset or completion of therapy or in cases of disease recurrence) is usually done by means of CT or combined ${ }^{18} \mathrm{~F}$-fluoro-2-deoxy-Dglucose positron emission tomography FDG-PET/CT [7, 8]. FDG-PET/CT has a central role in the management of childhood HD. However, FDG-PET does not form part of the standard imaging protocol in childhood NHL, although it is often performed in an ad hoc fashion. However, a disadvantage of FDG-PET/CT is the patient's exposure to ionizing radiation that may lead to the development of second neoplasms in later life [9]. This is especially important in children and adolescents, because they are inherently more radiosensitive than adults, and because they have more remaining years of life during which a radiation-induced cancer could develop [9].

Over the past decades outcome of children with malignant lymphoma has improved considerably, with long-term event-free survival rates of $>90 \%$ in childhood HD and $>80 \%$ in childhood NHL $[10,11]$. Current treatment strategies for malignant lymphoma aim at maximizing the chance of cure, while minimizing (late) toxicity such as infertility, premature menopause, cardiac disease, and most importantly, risk of second neoplasms $[10,11]$. Therefore, addressing the radiation risks from CT is an important issue in this population. Using dedicated 
low-dose CT protocols in children may reduce the risk of radiation-induced fatal cancer from $\mathrm{CT}$ [12]. An alternative solution is to replace FDG-PET/CT by other radiation-free imaging modalities, such as US or MRI [12]. In the context of staging of malignant lymphoma, whole-body MRI may be an excellent alternative to FDG-PET/CT, because it allows cross-sectional imaging of the entire body, provides a high soft-tissue contrast, and offers a wide arsenal of anatomical and functional sequences. With regard to the latter, whole-body DWI has recently been introduced as a potentially useful functional imaging modality to evaluate malignant lymphomas $[13,14]$. Since some centres already perform MRI for staging, DWI may be added to the routine MRI protocol as a potentially valuable adjunct. In this article, whole-body MRI techniques for staging malignant lymphoma, and whole-body DWI in particular, will be described and illustrated. Furthermore, future considerations and challenges in whole-body DWI will be discussed.

\section{Whole-body MRI techniques for staging malignant lymphoma}

\section{Conventional whole-body MRI}

Although there is no standard protocol for whole-body MRI, and the definition of "whole-body MRI" is rather arbitrary, it is usually performed or displayed in the coronal plane, with a field of view (FOV) that provides coverage of at least the area from the head/neck to the upper thighs (Figs. 1 and 2) [15-18]. Previously, separate body regions were imaged by repeatedly repositioning the patient [19]. However, this approach was time-consuming and uncomfortable for both the examiner and the patient. At present, this limitation has been overcome by the widespread availability of sliding table platforms that allow sequential movement of the patient through the bore of the magnet without patient repositioning [20]. Commonly applied sequences for whole-body MRI include (contrast-medium enhanced) T1-weighted (T1-W) and (fat-suppressed) T2weighted (T2-W) imaging, but there is no consensus yet on which combination of sequences provides the highest diagnostic accuracy while being time-efficient. Nonetheless, previous studies have shown the particular utility of short inversion time inversion recovery (STIR) whole-body MRI for staging malignant lymphoma [16-18].

STIR is a sensitive method for the detection of parenchymal and bone marrow lesions, that are generally highlighted as high signal intensity structures on this sequence [16-18, 21]. However, malignant lymph nodes cannot be differentiated from non-malignant nodes on the basis of signal intensity yet, neither on T1-W nor T2-W sequences. Thus, assessment of nodal involvement is still based on size criteria on conventional MRI. So far, only three studies have investigated the value of conventional whole-body MRI for the staging of malignant lymphoma [16-18]. Brennan et al. [16] performed a study in 23 adults that presented either for initial staging or restaging of HD or NHL and reported that whole-body MRI using STIR enables disease staging and that it compared favourably with CT for the detection of lymph nodes $>12 \mathrm{~mm}$ in short-axis diameter and for the detection of bone marrow involvement. Kellenberger et al. [17] performed a small study in eight children for initial staging and restaging of HD or NHL and reported that whole-body MRI using STIR is a sensitive technique for staging malignant lymphoma and that it is superior to blind bone marrow biopsy and conventional imaging (including CT, gallium-67 scintigraphy and bone scintigraphy) for the detection of bone marrow involvement at initial diagnosis. However, whole-body MRI using STIR was reported to lack specificity for diagnosing recurrent or residual disease [17]. Finally, Kwee et al. [18] compared whole-body MRI using both T1-W and STIR sequences to CT for the initial staging of 31 patients with HD or NHL. Staging results of whole-body MRI were equal to those of CT in $74 \%(23 / 31)$, higher in $26 \%(8 / 31)$, and lower in $0 \%$ $(0 / 31)$ of patients, with correct/incorrect/unresolved overstaging relative to $\mathrm{CT}$ in 3,2 and 1 patient(s) respectively, and incorrect staging of both modalities in 1 patient [18]. Results of these studies suggest that staging of malignant lymphoma using whole-body MRI is feasible and promising, but definitive conclusions regarding its value in the diagnostic management of malignant lymphoma cannot be made yet [16-18].

\section{Rationale for DWI}

A disadvantage of conventional (T1- or T2-W) whole-body MRI is the large amount of data that has to be evaluated, including data from many normal structures such as fat, muscles and vascular structures. Consequently, image interpretation can be time-consuming and subtle lesions may be overlooked. DWI, also known as intravoxel incoherent motion imaging, may overcome this disadvantage of conventional whole-body MRI. The most common approach to render MRI sensitive to diffusion is by using a spin-echo sequence and placing two strong gradients [socalled motion-probing gradients (MPGs)] on either side of the $180^{\circ}$ refocusing pulse [22]. DWI is basically a T2-W sequence, but the application of two strong MPGs results in a decrease in signal intensity of all structures. Importantly, the amount of signal intensity decrease induced by the MPGs is not similar for all structures, but depends on the degree of apparent diffusion that occurs between the MPGs; structures with a relatively low diffusivity are less sup- 


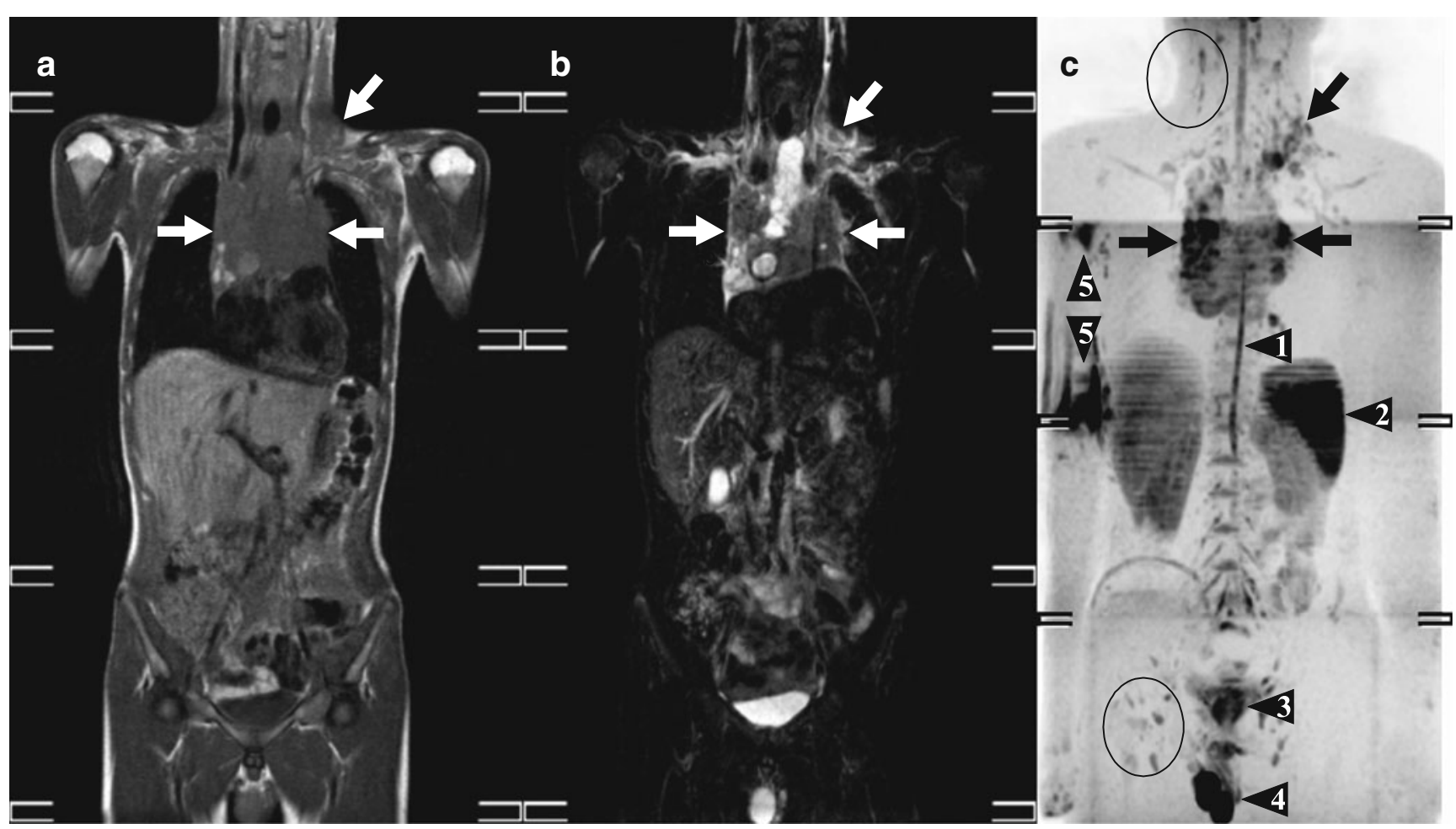

Fig. 1 15-year-old boy with stage II nodular sclerosing Hodgkin disease. Coronal whole-body (a) T1-W, (b) STIR and (c) greyscale inverted MIP DWI show cervical and mediastinal lymph node involvement (arrows). Note normal high signal intensity of the spinal cord (arrowhead 1),

pressed than tissues with a relatively high degree of diffusion or perfusion (e.g. vascular structures, cerebrospinal fluid and urine). Since most lesions (both benign and malignant) exhibit a relatively impeded diffusion, they generally lose less signal than surrounding normal background structures at DWI, resulting in a high lesion-tobackground contrast. Furthermore, fat suppression [that is inherently necessary in echo-planar imaging (EPI) to avoid image degradation due to severe chemical shift] also improves lesion-to-background contrast. Thus, DWI may increase lesion detection rate while decreasing image interpretation time. As mentioned previously, DWI is basically a T2-W sequence; consequently, residual T2 components (also known as T2 shine-through) can be seen on DWI that may mimic areas of impeded diffusion. For this reason, it is often recommended to review a corresponding apparent diffusion coefficient (ADC) map that does not contain any residual T2 components and allows quantification of diffusion; this is especially important in diagnosing acute ischaemic stroke [23]. However, in order to create an ADC map, at least two datasets with different degrees of diffusion-weighting (i.e. b-values) have to be acquired. Furthermore, lesion-to-background contrast on the ADC map is poor, and misregistration of the different datasets obtained with different $b$-values may occur (e.g. due to patient motion or image distortion). spleen (arrowhead 2), prostate (arrowhead 3) and testes (arrowhead 4) at whole-body DWI. Also note insufficiently suppressed fat (arrowheads 5), not to be mistaken for pathologic lesions. Normal lymph nodes (encircled) are also visualized at whole-body DWI

Moreover, and perhaps more importantly, T2 shine-through should not always be regarded as a disadvantage. In fact, many lesions exhibit both a prolonged $\mathrm{T} 2$ value and an impeded diffusion; both components contribute to the high conspicuity of lesions at DWI.

\section{DWI: from the brain to the body}

Since the mid-1990s, DWI has developed into a well-accepted method for the diagnosis of acute ischaemic stroke [23]. Of note, in acute ischaemic stroke apparent diffusion is (most likely) impeded due to cytotoxic oedema that gives high signal at DWI $[23,24]$. Previously, DWI outside the brain was not routinely feasible because the use of EPI, that is necessary for ultrafast imaging, could lead to severe image distortion in the magnetically inhomogeneous body. This limitation has relatively recently been overcome by the development of stronger and faster gradients, and, most importantly, parallel acquisition techniques that allow shortening of the echo-train length in EPI, thereby reducing image distortion [25].

Whole-body DWI with background body signal suppression: DWIBS

It was previously thought that diffusion-weighted contrast could not be maintained during bulk tissue motion and, in 


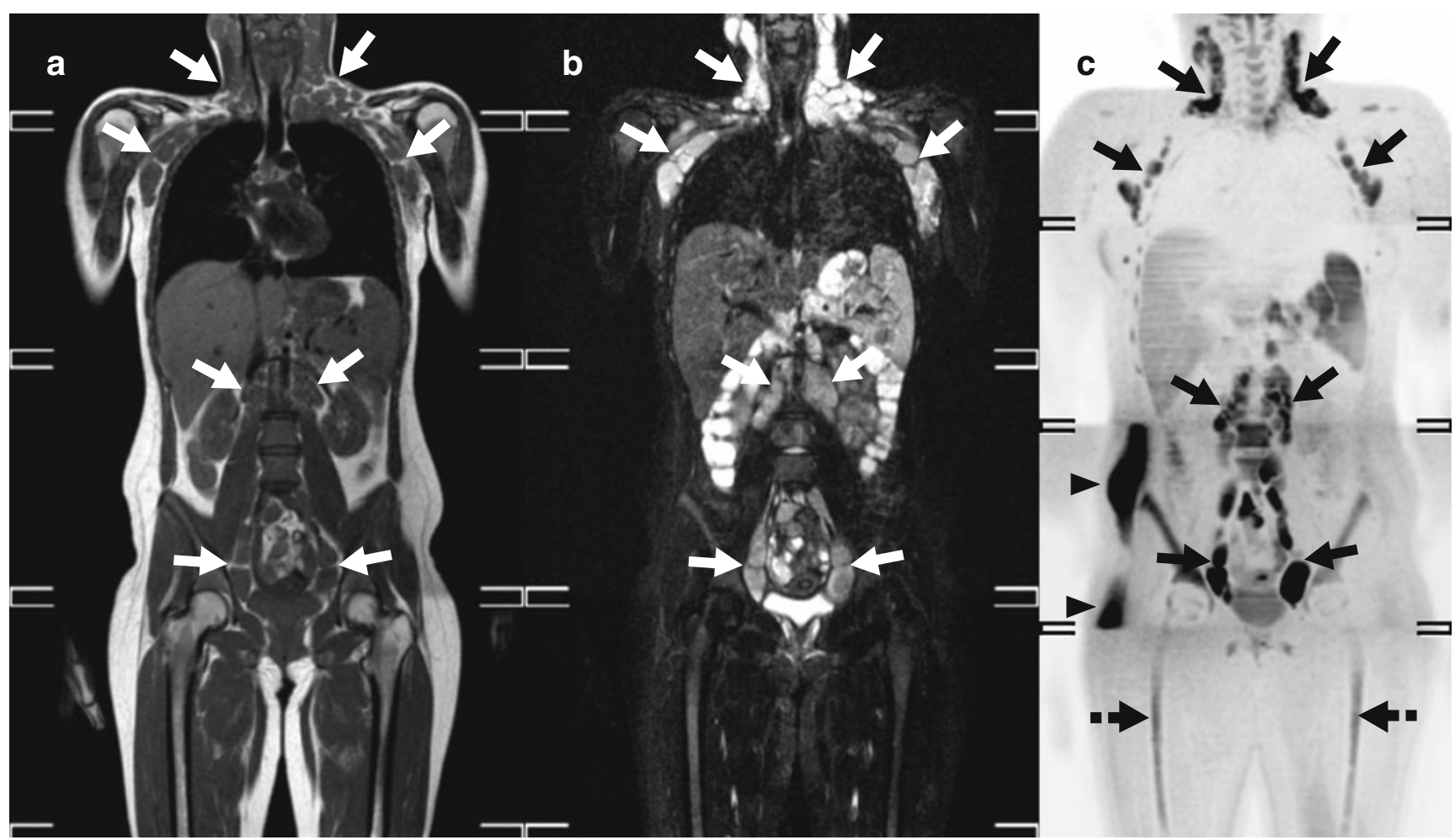

Fig. 2 17-year-old girl with unspecified widespread lymphoproliferative disease. Coronal whole-body (a) T1-W, (b) STIR and (c) greyscale inverted DWI show extensive (bilateral) cervical, axillary, paraaortic, mesenteric, and pelvic lymph node involvement (continuous arrows).
Note relatively high signal intensity of the bone marrow (e.g. in both femoral diaphyses (dashed arrows) suggestive of bone marrow hyperplasia (reconversion). Also note insufficiently suppressed fat in the right flank and buttock (arrowheads) particular, respiratory motion because diffusion takes place over several micrometres during the period in which the MPGs are applied, whereas respiratory motion takes place on the order of centimetres. Thus, breath-hold or respiratory-triggered DWI was considered necessary for DWI of the chest and abdomen. However, in breath-hold DWI, only thick slices (usually $8-10 \mathrm{~mm}$ ) with relatively low signal-to-noise ratio (SNR) can be obtained, that cannot be used to create multiplanar reformats (MPRs) or 3-D displays such as maximum intensity projections (MIPs).

Respiratory-triggered DWI does not have these disadvantages, but can considerably prolong the scan time. Thus, both breath-hold and respiratory-triggered DWI are not suitable for a whole-body DWI examination. A major breakthrough for whole-body DWI was the proof of the feasibility of DWI under free-breathing [13, 14]. The fact that a free-breathing acquisition is feasible can be explained by the fact that respiratory motion can be regarded as coherent motion during the period in which the MPGs are applied, and as such does not affect intravoxel incoherent motion (i.e. diffusion) [13, 14]. The concept of DWI under free-breathing was called diffusion-weighted whole-body imaging with background body signal suppression (DWIBS). The free-breathing approach allows thin slices (typically $4-5 \mathrm{~mm}$ ) with multiple signal averages (to increase SNR) to be obtained in a practically acceptable scan time and the acquired dataset can be used to create multiplanar reformats and 3-D displays. Furthermore, in DWIBS, high b-values (typically $1000 \mathrm{~s} / \mathrm{mm}^{2}$ ) are applied to ensure sufficient suppression of background body signals while highlighting lesions. Inverting the greyscale of DWIBS images gives them the typical appearance of PET-like images (Figs. 1, 2, 3, 4). Further details about the concept of DWIBS, including its practical implementation and parameter settings, can be read elsewhere [14].

Currently, a whole-body DWI examination can be performed in approximately 20-30 min. When adding conventional (T1- and T2-W) whole-body MRI, total examination time can be approximately $45 \mathrm{~min}$. Suggested sequences for whole-body MRI/DWI at 1.5-T are listed in Table 1. In our experience, children aged 8 years and older tolerate such an examination very well, without the need for any sedation [of note, most childhood malignant lymphomas occur in older children and adolescents (2)]. One recent study compared a combination of conventional whole-body MRI (T1-W and STIR sequences) and wholebody DWI (without using any ADC mapping) to CT for the staging of 28 patients with newly diagnosed HD or NHL [18]. Staging results of combined conventional whole-body MRI and whole-body DWI were equal to those of CT in $75 \%(21 / 28)$, higher in $25 \%(7 / 28)$, and lower in $0 \%(0 / 28)$ 


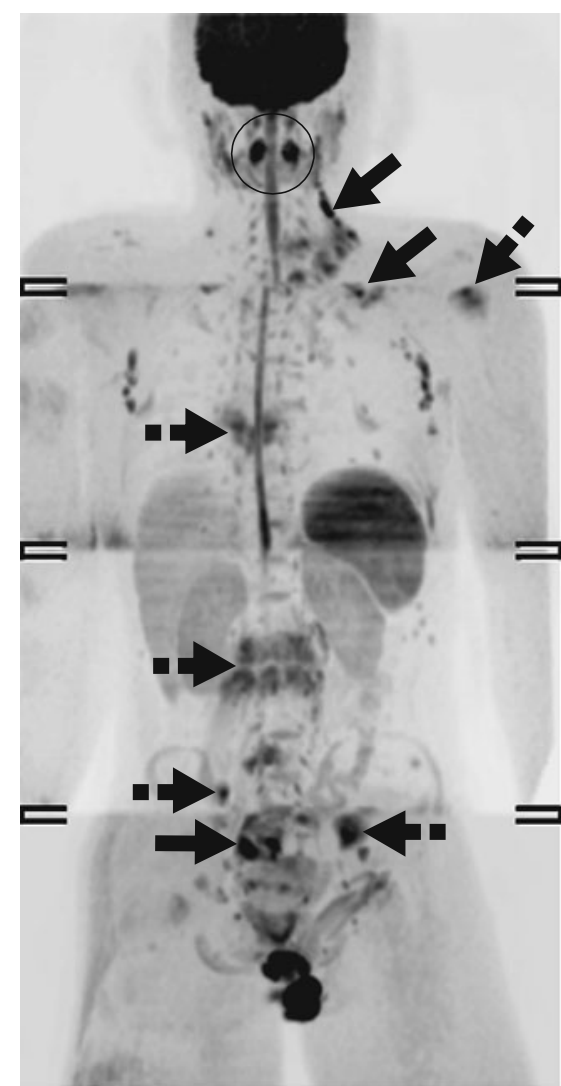

Fig. 3 16-year-old boy with stage IV nodular sclerosing Hodgkin disease. Coronal whole-body greyscale inverted MIP DWI shows left cervical, infraclavicular, and pelvic lymph node involvement (continuous arrows), and left humeral, vertebral, and pelvic bone marrow involvement (dashed arrows). Note that both pharyngeal tonsils (encircled) also exhibit high signal intensity, but this is a normal finding

of patients, with correct/incorrect overstaging relative to CT in 6 and 1 patient(s), respectively. Interestingly, the combination of conventional whole-body MRI with whole-body DWI correctly upstaged 4 of 28 patients compared to conventional whole-body MRI alone, that well reflects the potential additional diagnostic value of DWI [18]. Nevertheless, although these initial results appear very promising compared to $\mathrm{CT}$, more studies with larger sample sizes are needed. Furthermore, whole-body DWI has not yet been compared to FDG-PET/CT.

\section{Whole-body DWI for staging malignant lymphoma; specific considerations}

Nodal pathology

One of the first observations made when DWI was applied in the entire body was the clear visualization of lymph nodes, that appear as high signal intensity structures, while surrounding background tissues (including fat) are suppressed (Figs. 1-4) [13]. For this reason it was thought that

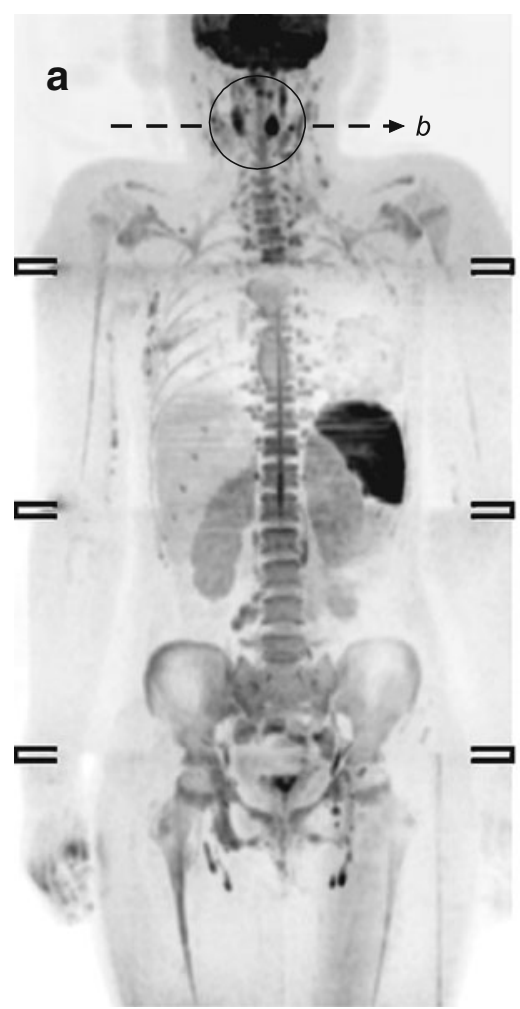

b

Fig. 4 12-year-old girl with stage I diffuse large B-cell lymphoma arising in the pharyngeal tonsils (histologically proven). Coronal whole-body greyscale inverted MIP DWI (a) shows no obvious nodal or extranodal pathology, except for striking high signal intensity in both pharyngeal tonsils (encircled). Although the normal Waldeyer ring often exhibits high signal intensity at DWI (also shown in Fig. 3), the corresponding axial image (b) shows size asymmetry of the pharyngeal tonsils, indicating lymphomatous involvement

one promising application of whole-body DWI could be in staging of malignant lymphoma. However, one important limitation of DWI is that it visualizes both non-malignant and malignant lymph nodes (Fig. 1). This is because normal lymph nodes already have a relatively low diffusivity (probably due to high cellularity) and long T2 value. Several studies have reported that lymphomatous lymph nodes have lower ADCs than metastatic lymph nodes or benign lymphadenopathy (probably due to increased cellularity). However, these studies only included pathologically enlarged lymph nodes without considering (normal-sized) "healthy" lymph nodes [26-30]. Therefore, the value of ADC measurements in discriminating (normal-sized) "healthy" lymph nodes from (normal-sized) lymphomatous lymph nodes is still unknown. In addition, one recent study performed an inter- and intra-observer reproducibility study regarding $\mathrm{ADC}$ measurements of normal-sized lymph nodes [31]. The ranges of mean ADC difference \pm limits of agreement (in $10^{-3} \mathrm{~mm}^{2} / \mathrm{s}$ ) for inter-observer agreement were $-0.03-0.02 \pm 0.15-0.31$. In addition, ranges of mean ADC difference \pm limits of agreement (in $10^{-3} \mathrm{~mm}^{2} / \mathrm{s}$ ) for intra-observer agreement were $0.00-0.04 \pm 0.13-0.32$ [31]. 
Table 1 Suggested sequences for whole-body MRI/DWI at 1.5-T. SFR spectral fat saturation. ${ }^{1}$ DWI can be combined with either spectral fat saturation (DWI-SFS) or a STIR pre-pulse (DWI-STIR) for fat suppression. DWI-SFS offers higher SNR and is relatively less time-consuming than DWI-STIR. However, DWI-STIR offers more robust fat suppression over an extended FOV than DWI-SFS, because STIR is less sensitive to magnetic field inhomogeneities. Because the head, neck and shoulder regions usually suffer from considerable magnetic field inhomogeneities, it is recommended to use DWI-STIR for these body regions. In addition, DWI-STIR may be useful in suppressing bowel signal that often has a short $\mathrm{T} 1$ relaxation time, similar to that of fat. ${ }^{2}$ At present, it is recommended to perform DWI in the axial plane and to coronally reformat the acquired dataset afterwards, because direct coronal scanning may still suffer from considerable image distortion due to the need for a larger FOV. ${ }^{3} \mathrm{~A} \mathrm{~b}$ value of $1000 \mathrm{~s} / \mathrm{mm}^{2}$ is effective in suppressing background body signals while highlighting lesions throughout the entire body. Therefore, a b-value of $1000 \mathrm{~s} / \mathrm{mm}^{2}$ can be recommended for wholebody imaging. The relatively low signal at a b-value of $1000 \mathrm{~s} / \mathrm{mm}^{2}$ can be increased by acquiring multiple signal averages. The additional acquisition of a b-value of $0 \mathrm{~s} / \mathrm{mm}^{2}$ allows quantitative diffusion measurements. ${ }^{4}$ Effective scan time without taking into account the time needed for acquiring survey scans, breath holding, respiratory gating, table movements, and coil repositioning. ${ }^{5}$ Using DWI-SFS for three stations covering the pelvis, abdomen and chest, and using DWISTIR for the station that covers the head, neck, and shoulder

\begin{tabular}{|c|c|c|c|c|}
\hline \multirow{2}{*}{$\begin{array}{l}\text { Parameter } \\
\text { Pulse sequence } \\
\text { Repetition time (ms) }\end{array}$} & \multicolumn{2}{|c|}{$\begin{array}{l}\text { T1-W } \\
\text { Single-shot turbo spin-echo }\end{array}$} & \multicolumn{2}{|c|}{$\begin{array}{l}\text { DWI-SFS }^{1} \quad \text { DWI-STIR }^{1} \\
\text { Single-shot spin-echo } \\
\text { echo-planar imaging }\end{array}$} \\
\hline & 537 & 2444 & 6962 & 8612 \\
\hline Echo time (ms) & 18 & 64 & 78 & \\
\hline Inversion time (ms) & - & 165 & 180 & - \\
\hline Receiver bandwidth $(\mathrm{Hz})$ & 460.1 & 487.9 & 30.2 & \\
\hline Slice orientation & Coronal & & Axial $^{2}$ & \\
\hline Slice thickness $(\mathrm{mm})$ & 6.0 & & 4.0 & \\
\hline Slice gap $(\mathrm{mm})$ & 1.0 & & 0.0 & \\
\hline No. of slices per station & 30 & & 60 & \\
\hline Cranio-caudal coverage per station (mm) & 265 & & 240 & \\
\hline Field of view $\left(\mathrm{mm}^{2}\right)$ & $530 \times 265$ & $530 \times 265$ & $450 \times 360$ & \\
\hline Acquisition matrix & $208 \times 287$ & $336 \times 120$ & $128 \times 81$ & \\
\hline Directions of motion probing gradients & - & & Phase, frequ & , and slice \\
\hline B-values $\left(\mathrm{s} / \mathrm{mm}^{2}\right)$ & - & & 0 and $1000^{3}$ & \\
\hline No. of signals averaged & 1 & 2 & 3 & \\
\hline Partial Fourier acquisition (\%) & - & & 0.651 & \\
\hline Parallel acceleration factor & - & & 2 & \\
\hline Echo-planar imaging factor & - & & 43 & \\
\hline Respiratory motion compensation techniques & $\begin{array}{l}\text { Breath holding o } \\
\text { when scanning }\end{array}$ & $\begin{array}{l}\text { gating are recommended } \\
\text { abdomen }\end{array}$ & $\begin{array}{l}\text { Image acqui } \\
\text { free breath }\end{array}$ & n under \\
\hline Acquired voxel size $\left(\mathrm{mm}^{3}\right)$ & $1.27 \times 1.85 \times 6.00$ & $1.58 \times 2.21 \times 6.00$ & $3.52 \times 4.50 \times$ & \\
\hline Reconstructed voxel size $\left(\mathrm{mm}^{3}\right)$ & $1.04 \times 1.04 \times 6.00$ & $1.04 \times 1.04 \times 6.00$ & $1.76 \times 1.76 \times$ & \\
\hline Effective scan time per station ${ }^{4}$ & $47 \mathrm{~s}$ & $44 \mathrm{~s}$ & $3 \min 20 \mathrm{~s}$ & $4 \min 4 \mathrm{~s}$ \\
\hline Total no. of stations & 7 & & $3(+1)^{5}$ & 4 \\
\hline Total effective scan time ${ }^{4}$ & $5 \min 48 \mathrm{~s}$ & $5 \min 13 \mathrm{~s}$ & $14 \min 4 s^{5}$ & $16 \min 26 s$ \\
\hline
\end{tabular}

Given this relatively large variability, inter- and intra-observer reproducibility of ADC measurements of normal-sized lymph nodes appears to be rather poor. Thus, the detection of lymphomatous lymph nodes in whole-body DWI is still based on size criteria. Nonetheless, compared to conventional (T1and T2-W) whole-body MRI, whole-body DWI provides a faster and more straightforward evaluation of the amount and distribution of (pathologically enlarged) lymphomatous lymph nodes.

There are some potential pitfalls and drawbacks that have to be taken into account when evaluating whole-body DWI with respect to the evaluation of nodal/lymphoid pathology. First DWI, and in particular DWIBS (that is used for whole-body DWI), highlights lymph nodes while suppressing surrounding normal structures. Because of the lack of anatomical reference, size measurements of lymph nodes at DWI are highly dependent on the applied window level and window width. In addition, there are no published studies comparing nodal measurements at DWI with those at conventional imaging (either CT or T1- and T2-W images). Thus, at present it is recommended that sizes of lymph nodes that are suspicious for malignancy at DWI are measured on conventional $\mathrm{T} 1-$ or $\mathrm{T} 2-\mathrm{W}$ images for verification. Nevertheless, the efficacy of such an approach 
is still unknown. Another issue is that, unlike respiratory motion, cardiac motion may result in non-rigid body motion and signal loss of structures close to the heart [32, 33]. Consequently, the evaluation of mediastinal lymph nodes may be affected by cardiac motion. Nevertheless, relatively large mediastinal masses can readily be identified at DWI (Fig. 1).

\section{Extranodal pathology}

DWI is a very sensitive method for the detection of extranodal lymphomatous lesions that generally exhibit high signal intensity at DWI (due to low diffusivity and long T2) (Fig. 3). Importantly, several benign conditions (including inflammatory and infectious lesions) also have high signal intensity at DWI. To minimize the number of false-positive results DWI should be interpreted along with other sequences and patient history and findings on clinical examination should be known when interpreting the images. Although there are as yet no established criteria for classifying lesions as negative or positive for lymphomatous involvement, a signal intensity higher than that of the spinal cord has often been proposed as suggestive of malignancy [33-36]. On the other hand, diffusivity may be increased in cases of necrosis (provided no haemorrhagic components are present), that can be visualized as areas of relatively low signal intensity at DWI.

Another important issue is that several normal extranodal structures (including brain, salivary glands, Waldeyer ring, thymus, spleen, gallbladder, adrenal glands, prostate, testes, penis, endometrium, ovaries, spinal cord, peripheral nerves, and bone marrow) (may) also exhibit high signal intensity at DWI, due to their relatively low diffusivity and long T2 value (Fig. 1) [13, 14]. In our experience, Waldeyer ring in particular may appear very hyperintense at DWI (Figs. 3, 4). Detection of abnormality in this organ based on differences in signal intensity may be difficult, although an asymmetrical appearance may suggest the presence of lymphomatous involvement (Fig. 4). Another lymphoid organ that often appears very hyperintense at DWI is the spleen (Figs. 1-4). Consequently, abnormality in this organ may be obscured. On the other hand, visibility of the spleen is variable and probably dependent on its iron content (and consequent signal loss). Similarly, the visibility of the liver is related to its iron content, although this organ usually appears relatively hypointense at high b-values (i.e. $\geq 1000 \mathrm{~s} / \mathrm{mm}^{2}$ ). A potential pitfall in DWI is in the evaluation of the bone marrow. During skeletal maturation, haematopoietic (red) marrow is converted to fatty (yellow) marrow [37]. At DWI, yellow marrow appears hypointense thanks to the use of fat suppression. Red bone marrow appears hyperintense at DWI, probably due to its high cellularity that has a relatively impeded diffusion [38].
Residual or reconverted red bone marrow (islands) may resemble lymphomatous bone marrow involvement (Fig. 2). Thus, signal intensities of normal and lymphomatous bone marrow may overlap at DWI [39]. Nevertheless, additional conventional (T1- or T2-W) MRI sequences may increase the diagnostic performance of DWI alone in the assessment of the bone marrow. Despite its imperfect specificity, DWI may have a role in ruling out bone marrow involvement and guiding bone marrow biopsy $[39,40]$.

Other important body regions that require special consideration are the liver and lung. Similar to bone marrow involvement, liver or lung involvement, which are not uncommon in malignant lymphoma, represent stage IV disease [3, 4, 41, 42]. Usually, DWI of the liver is acquired using breath-holding or respiratory triggering [43]. In particular, the suppression of liver vessels in DWI is an advantage over conventional MRI sequences and increases sensitivity for the detection of lesions [44]. However, as mentioned previously, whole-body DWI is acquired using free-breathing. Despite the fact that the breathing approach introduces slight image blurring due to diaphragmatic motion, liver lesions can generally be well depicted thanks to thin slice acquisition and the high degree of signal averaging [45].

DWI of the lung is a particular challenge; besides cardiac and respiratory motion artefacts, susceptibility-induced image distortion and signal loss due to the many air-tissue interfaces may occur. Nevertheless, although sensitivity of CT still outperforms that of MRI/DWI in the lung, studies in patients with pulmonary nodules have shown that DWI is able to detect lung lesions up to $5 \mathrm{~mm}$ in diameter [34-36]. Moreover, unlike CT, DWI is able to provide functional tissue information that may aid in the differentiation between benign and malignant pulmonary lesions [34-36]. Of note, DWI has been shown to diagnostically outperform FDG-PET in the characterization of pulmonary nodules/ masses [36]. Despite its promise, the diagnostic performance of DWI in the detection of lymphomatous lung lesions requires further investigation.

\section{Role of imaging beyond staging}

Imaging plays a crucial role in staging of malignant lymphoma, but may also be of value in the (early) assessment of response to therapy. Early assessment of response to therapy will allow more individualized therapy that will maximize the chance of cure while minimizing risk of (late) toxicity. However, anatomic imaging modalities such as CT, US and conventional MRI sequences may not be sufficiently accurate in discriminating residual disease from fibrosis or scar tissue [46-48]. FDG-PET, on the other hand, provides functional tissue information, and 
may be superior to conventional imaging modalities in this context. A recent study by Furth et al. [48] has indeed shown that FDG-PET is superior to conventional imaging methods (i.e. contrast-enhanced MRI of neck, abdomen, and pelvis; US examinations of all lymph node regions; as well as contrast-enhanced CT of the chest) with regard to specificity in early (i.e. after two cycles of chemotherapy) and late response assessment (i.e. after completion of chemotherapy) in children with HD (68\% versus $3 \%$, and $78 \%$ versus $11 \%$, respectively; both $P<0.001)$. Specificity of early therapy response assessment by FDG-PET was improved to $97 \%$ by quantitative analysis of maximal standardized uptake value reduction using a cut-off value of $58 \%$. These data suggest that FDG-PET should not be omitted either for staging or response assessment [48]. At present it is still highly speculative whether DWI can replace FDG-PET in the diagnostic management of malignant lymphoma. Interestingly, a voxel-based quantitative DWI approach [functional diffusion mapping, currently referred to as parametric response mapping of diffusion $\left.\left(\mathrm{PRM}_{\mathrm{ADC}}\right)\right]$ has recently shown promise to give an early prediction of survival in patients with high-grade gliomas $[49,50]$. However, whole-body quantitative DWI techniques still have to be developed and subsequently compared to the current functional imaging modality of choice in malignant lymphoma; FDG-PET.

\section{Future considerations and challenges}

\section{Reducing image blurring}

Diffusion-weighted contrast is maintained under free breathing acquisition (i.e. DWIBS), although at the expense of slight image blurring. Image blurring may affect interpretation of paradiaphragmatic organs, such as the liver. An effective approach to reduce image blurring in DWIBS may be the use of a navigator echo technique that allows continuous real-time slice tracking and position correction, without the use of any gating window. Consequently, image blurring is reduced without considerably prolonging scan time. Implementing such a technique in a whole-body DWI sequence for staging malignant lymphoma may be attractive, since it can improve visualization of peridiaphragmatic organs $[45,51]$.

\section{Whole-body DWI at 3.0-T}

Whole-body DWI is currently best performed at 1.5-T, using a phased-array receiver surface coil. Nevertheless, SNR in DWI is relatively low, even though a high number of signal averages can be acquired when using the concept of DWIBS. SNR is increased when performing DWI at higher field strength, since SNR increases linearly with field strength [52]. This in turn may improve staging accuracy in malignant lymphoma. A recent feasibility study showed that DWIBS at 3.0-T offers higher SNR compared to that at 1.5-T, but the former still suffers from a higher risk of $\mathrm{B} 0$ and $\mathrm{B} 1$ inhomogeneities and susceptibility artefacts [53]. Therefore, the further development of multi-source radiofrequency (RF) transmission technology (that will be discussed later) and non-EPI-based DWI techniques is important to solve these problems $[54,55]$. Another challenge at high field strength is fat suppression; inadequately suppressed fat may hinder image interpretation. Compared to a frequency selective (spectral fat saturation) pre-pulse, a short inversion time inversion recovery (STIR) pre-pulse has been shown to offer the best fat suppression in all body regions at 3.0-T, since the latter is less sensitive to B0 inhomogeneities [53]. However, the use of STIR inherently yields lower SNR than the use of spectral fat saturation [56]. Furthermore, both spectral fat saturation and STIR require additional RF and/or gradient pulses, thereby increasing the specific absorption rate (SAR) and prolonging scan time [56].

Thus, the development of new fat suppression techniques is important to exploit the full potential of whole-body DWI at higher field strength. In this respect, the reintroduction of the slice-selection gradient reversal (SSGR) technique is very promising $[57,58]$. The SSGR technique uses two sliceselection gradients of opposing polarity for the $90^{\circ}$ excitation pulse and the $180^{\circ}$ refocusing pulse in a spin-echo EPI sequence for diffusion sensitization. The SSGR technique exploits the high sensitivity of EPI to chemical shift artefacts (water-fat shift) in the phase-encoding direction due to the relatively low bandwidth in that orientation; water signal will be completely preserved, but only fat signal that was exposed to both the excitation pulse and the refocusing pulse in the centre of the water slice will contribute to image formation. Since chemical shift artefacts are more pronounced at higher field strengths, fat signal can be significantly reduced or even completely eliminated using the SSGR technique at field strengths $\geq 3$. 0 -T. Furthermore, unlike spectral fat saturation and STIR, the SSGR technique does not prolong scan time, nor does it increase SAR [57, 58].

Another promising development that was recently implemented at a clinical 3.0-T system is the multi-source RF transmission technology [54]. Using this technology, independent RF pulses are sent; this improves image uniformity and consistency compared to single-source RF transmission that may suffer from dielectric resonance effects at high field strength. Furthermore, local RF deposition (SAR) can be reduced, allowing for faster scanning [54]. Thus, although 1.5-T systems still provide the best and most reproducible image quality, eventually it is expected that whole-body DWI at 3.0-T will become possible in routine clinical practice. 
Shifting gears in whole-body molecular imaging

The discrimination between normal-sized non-malignant lymph nodes and normal-sized lymphomatous lymph nodes is still an unsolved issue in DWI. This is because both normal and malignant lymph nodes are highlighted at DWI, and ADC measurements have not yet been shown to discriminate [31]. Ultrasmall superparamagnetic iron oxide (USPIO)-enhanced MRI was introduced in the early 1990s as a promising imaging modality for evaluating lymph nodes, allowing for the identification of malignant nodal infiltration independent of lymph node size [59, 60]. Intravenously administered USPIOs are taken up by macrophages in the reticuloendothelial system, predominantly within the lymph nodes, but also in Waldeyer ring, spleen and bone marrow. Normal homogeneous uptake of USPIOs in nonmetastatic lymph nodes shortens the $\mathrm{T} 2$ and $\mathrm{T} 2 *$ values, turning these lymph nodes hypointense on T2- and $\mathrm{T} 2 *-\mathrm{W}$ images, whereas malignant lymph nodes lack uptake and remain hyperintense [60]. USPIO-enhanced lymphography has been shown to achieve higher diagnostic precision than conventional, unenhanced MRI for the detection of lymph nodes metastases of various tumours [61]. However, conspicuity and detectability of lymph nodes is relatively low in USPIO-enhanced MRI. Consequently, image interpretation can be very time consuming, and malignant lymph nodes of small size may be missed. Furthermore, one image dataset before and one image dataset after USPIO administration has to be obtained, that poses an extra burden on the patient, and on logistic and financial resources.

Performing DWI after USPIO administration may overcome the disadvantages of DWI and conventional (T2- or T2*-W) USPIO-enhanced MRI alone. Theoretically, in USPIO-enhanced DWI only malignant lymph nodes will be highlighted; the high lymph node-to-background contrast will reduce image interpretation time and will obviate the need to acquire an additional dataset of images before USPIO administration. The feasibility of this new concept has recently been proven in patients with urinary bladder and prostate cancer (although attention should be paid to possible oversuppression by USPIOs as a result of which micrometastases may be missed); an extension of this concept to the entire body (i.e. USPIO-enhanced whole-body DWI) may provide a highly accurate method for the determination of extent of nodal involvement in patients with malignant lymphoma [62]. An important additional advantage of USPIO-enhanced whole-body DWI over whole-body DWI alone is suppression of signal from the normal Waldeyer ring, spleen and normal or hyperplastic red bone marrow, against which lymphomatous lesions in these organs will theoretically be highlighted [63].
Another promise for the future is the development of whole-body PET/MRI systems [64]. Although several technological difficulties have to be solved in the design of a fully integrated whole-body PET/MRI system (including the issues of electromagnetic interference between the two systems and MRI-based attenuation correction), and high (development) costs have to be taken into account, the integrated use of whole-body DWI and FDG-PET may be of interest in (paediatric) patients with malignant lymphoma [64]. For example, FDG-PET may increase diagnostic performance of DWI alone in several areas, including (normal-sized) lymph nodes, spleen, and bone marrow [7, $65,66]$. Furthermore, FDG-PET may be a powerful adjunct to whole-body MRI/DWI in restaging malignant lymphoma; restaging performance of whole-body MRI/DWI is still unknown, but FDG-PET has already proven to be of value in diagnosing persistent or recurrent disease [7, 67, 68]. On the other hand, an area where DWI may clearly be of advantage compared to FDG-PET is the urinary tract. Normal FDG accumulation in the renal collecting system, ureters and bladder may limit the use of FDG-PET in the evaluation of renal, paraortic, and pelvic lesions, whereas DWI does not have this disadvantage [69]. Another advantage of DWI over (FDG) PET is its superior spatial resolution. Furthermore, although most childhood NHLs are FDG-avid, DWI allows visualization of certain subtypes of NHLs that are not FDG-avid [7, 8].

\section{Conclusion}

Whole-body MRI and whole-body DWI in particular, provides a radiation-free alternative for staging malignant lymphoma in children. Whole-body DWI may potentially facilitate image interpretation and increase sensitivity of conventional (T1- and T2-W) whole-body MRI alone. Nevertheless, there is a strong need for comparative studies with FDG-PET/CT before definitive conclusions can be made regarding its value in the diagnostic management of malignant lymphoma. Integrating whole-body DWI with other molecular imaging strategies, including USPIO-enhanced imaging and FDG-PET, is expected to further increase its staging performance in malignant lymphoma.

Acknowledgement This work was supported by ZonMw Program for Health Care Efficiency Research (grant number 80-82310-98-08012).

Open Access This article is distributed under the terms of the Creative Commons Attribution Noncommercial License which permits any noncommercial use, distribution, and reproduction in any medium, provided the original author(s) and source are credited. 


\section{References}

1. Jemal A, Siegel R, Ward E et al (2009) Cancer statistics, 2009. CA Cancer J Clin 59:225-249

2. Steliarova-Foucher E, Stiller C, Kaatsch P et al (2004) Geographical patterns and time trends of cancer incidence and survival among children and adolescents in Europe since the 1970s (the ACCIS project): an epidemiological study. Lancet 364:2097-2105

3. Connors JM (2005) State-of-the-art therapeutics: Hodgkin's lymphoma. J Clin Oncol 23:6400-6408

4. Armitage JO (2005) Staging non-Hodgkin lymphoma. CA Cancer $\mathrm{J}$ Clin 55:368-376

5. Murphy SB (1980) Classification, staging and end results of treatment of childhood non-Hodgkin's lymphomas: dissimilarities from lymphomas in adults. Semin Oncol 7:332-339

6. Murphy SB, Fairclough DL, Hutchison RE et al (1989) NonHodgkin's lymphomas of childhood: an analysis of the histology, staging, and response to treatment of 338 cases at a single institution. J Clin Oncol 7:186-193

7. Kwee TC, Kwee RM, Nievelstein RA (2008) Imaging in staging of malignant lymphoma: a systematic review. Blood 111:504-516

8. Juweid ME, Stroobants S, Hoekstra OS et al; Imaging Subcommittee of International Harmonization Project in Lymphoma (2007) Use of positron emission tomography for response assessment of lymphoma: consensus of the Imaging Subcommittee of International Harmonization Project in Lymphoma. J Clin Oncol 25:571-578

9. Brenner DJ, Hall EJ (2007) Computed tomography-an increasing source of radiation exposure. N Engl J Med 357:2277-2284

10. Hodgson DC, Hudson MM, Constine LS (2007) Pediatric hodgkin lymphoma: maximizing efficacy and minimizing toxicity. Semin Radiat Oncol 17:230-242

11. Gross TG, Termuhlen AM (2007) Pediatric non-Hodgkin's lymphoma. Curr Oncol Rep 9:459-465

12. Semelka RC, Armao DM, Elias J Jr et al (2007) Imaging strategies to reduce the risk of radiation in $\mathrm{CT}$ studies, including selective substitution with MRI. J Magn Reson Imaging 25:900-909

13. Takahara T, Imai Y, Yamashita T et al (2004) Diffusion weighted whole body imaging with background body signal suppression (DWIBS): technical improvement using free breathing, STIR and high resolution 3D display. Radiat Med 22:275-282

14. Kwee TC, Takahara T, Ochiai R et al (2008) Diffusion-weighted whole-body imaging with background body signal suppression (DWIBS): features and potential applications in oncology. Eur Radiol 18:1937-1952

15. Schmidt GP, Baur-Melnyk A, Herzog P et al (2005) Highresolution whole-body magnetic resonance image tumor staging with the use of parallel imaging versus dual-modality positron emission tomography-computed tomography: experience on a 32channel system. Invest Radiol 40:743-753

16. Brennan DD, Gleeson T, Coate LE et al (2005) A comparison of whole-body MRI and CT for the staging of lymphoma. AJR 185:711-716

17. Kellenberger CJ, Miller SF, Khan M et al (2004) Initial experience with FSE STIR whole-body MR imaging for staging lymphoma in children. Eur Radiol 14:1829-1841

18. Kwee TC, Quarles van Ufford HM, Beek FJ et al (2009) WholeBody MRI, including diffusion-weighted imaging, for the initial staging of malignant lymphoma: comparison to computed tomography. Invest Radiol 44:683-690

19. Walker R, Kessar P, Blanchard R et al (2000) Turbo STIR magnetic resonance imaging as a whole-body screening tool for metastases in patients with breast carcinoma: preliminary clinical experience. J Magn Reson Imaging 11:343-350
20. Kavanagh E, Smith C, Eustace S (2003) Whole-body turbo STIR MR imaging: controversies and avenues for development. Eur Radiol 13:2196-2205

21. Kellenberger CJ, Epelman M, Miller SF et al (2004) Fast STIR whole-body MR imaging in children. Radiographics 24:1317-1330

22. Stejskal EO, Tanner JE (1965) Spin diffusion measurements: spin echoes in the presence of a time-dependent field gradient. J Chem Phys 42:288-292

23. Schaefer PW, Grant PE, Gonzalez RG (2000) Diffusion-weighted MR imaging of the brain. Radiology 217:331-345

24. Sevick RJ, Kanda F, Mintorovitch J et al (1992) Cytotoxic brain edema: assessment with diffusion-weighted MR imaging. Radiology 185:687-690

25. Pruessmann KP, Weiger M, Scheidegger MB et al (1999) SENSE: sensitivity encoding for fast MRI. Magn Reson Med 42:952-962

26. Holzapfel K, Duetsch S, Fauser C et al (2008) Value of diffusionweighted MR imaging in the differentiation between benign and malignant cervical lymph nodes. Eur J Radiol 72:381-387

27. King AD, Ahuja AT, Yeung DK et al (2007) Malignant cervical lymphadenopathy: diagnostic accuracy of diffusion-weighted MR imaging. Radiology 245:806-813

28. Abdel Razek AA, Soliman NY, Elkhamary S et al (2006) Role of diffusion-weighted MR imaging in cervical lymphadenopathy. Eur Radiol 16:1468-1477

29. Sumi M, Van Cauteren M, Nakamura T (2006) MR microimaging of benign and malignant nodes in the neck. AJR 186:749-757

30. Sumi M, Sakihama N, Sumi T et al (2003) Discrimination of metastatic cervical lymph nodes with diffusion-weighted MR imaging in patients with head and neck cancer. AJNR 24:1627-1634

31. Kwee TC, Takahara T, Luijten PR et al (2009) ADC measurements of lymph nodes: Inter- and intra-observer reproducibility study and an overview of the literature. Eur J Radiol Apr 15 [Epub ahead of print]

32. Norris DG (2001) Implications of bulk motion for diffusionweighted imaging experiments: effects, mechanisms, and solutions. J Magn Reson Imaging 13:486-495

33. Sakurada A, Takahara T, Kwee TC et al (2009) Diagnostic performance of diffusion-weighted magnetic resonance imaging in esophageal cancer. Eur Radiol 19:1461-1469

34. Uto T, Takehara Y, Nakamura Y et al (2009) Higher sensitivity and specificity for diffusion-weighted imaging of malignant lung lesions without apparent diffusion coefficient quantification. Radiology 252:247-254

35. Satoh S, Kitazume Y, Ohdama S et al (2008) Can malignant and benign pulmonary nodules be differentiated with diffusionweighted MRI? AJR 191:464-470

36. Mori T, Nomori H, Ikeda K et al (2008) Diffusion-weighted magnetic resonance imaging for diagnosing malignant pulmonary nodules/masses: comparison with positron emission tomography. $\mathrm{J}$ Thorac Oncol 3:358-364

37. Laor T, Jaramillo D (2009) MR imaging insights into skeletal maturation: what is normal? Radiology 250:28-38

38. Nonomura Y, Yasumoto M, Yoshimura R et al (2001) Relationship between bone marrow cellularity and apparent diffusion coefficient. J Magn Reson Imaging 13:757-760

39. Yasumoto M, Nonomura $Y$, Yoshimura R et al (2002) MR detection of iliac bone marrow involvement by malignant lymphoma with various MR sequences including diffusionweighted echo-planar imaging. Skeletal Radiol 31:263-269

40. Kwee TC, Kwee RM, Verdonck LF et al (2008) Magnetic resonance imaging for the detection of bone marrow involvement in malignant lymphoma. Br J Haematol 141:60-68

41. Baumhoer D, Tzankov A, Dirnhofer S et al (2008) Patterns of liver infiltration in lymphoproliferative disease. Histopathology 53:81-90

42. Berkman N, Breuer R, Kramer MR et al (1996) Pulmonary involvement in lymphoma. Leuk Lymphoma 20:229-237 
43. Naganawa S, Kawai H, Fukatsu H et al (2005) Diffusion-weighted imaging of the liver: technical challenges and prospects for the future. Magn Reson Med Sci 4:175-186

44. Parikh T, Drew SJ, Lee VS et al (2008) Focal liver lesion detection and characterization with diffusion-weighted MR imaging: comparison with standard breath-hold T2-weighted imaging. Radiology 246:812-822

45. Kwee TC, Takahara T, Ogino T et al (2009) Diffusion-weighted MR imaging (DWI) using TRacking only Navigator Echo (TRON): initial clinical evaluation and comparison to respiratory triggered and free breathing DWI. Presented at the ISMRM 17th Scientific Meeting \& Exhibition, Honolulu, Hawai'i, USA, April 18-24, 2009

46. Radford JA, Cowan RA, Flanagan M et al (1988) The significance of residual mediastinal abnormality on the chest radiograph following treatment for Hodgkin's disease. J Clin Oncol 6:940-946

47. Jerusalem G, Beguin Y, Fassotte MF et al (1999) Whole-body positron emission tomography using $18 \mathrm{~F}$-fluorodeoxyglucose for posttreatment evaluation in Hodgkin's disease and non-Hodgkin's lymphoma has higher diagnostic and prognostic value than classical computed tomography scan imaging. Blood 94:429-433

48. Furth C, Steffen IG, Amthauer H et al (2009) Early and late therapy response assessment with [18F] fluorodeoxyglucose positron emission tomography in pediatric Hodgkin's lymphoma: analysis of a prospective multicenter trial. J Clin Oncol 27:4385-4391

49. Hamstra DA, Galbán CJ, Meyer CR et al (2008) Functional diffusion map as an early imaging biomarker for high-grade glioma: correlation with conventional radiologic response and overall survival. J Clin Oncol 26:3387-94

50. Hamstra DA, Rehemtulla A, Ross BD (2007) Diffusion magnetic resonance imaging: a biomarker for treatment response in oncology. J Clin Oncol 25:4104-4109

51. Takahara T, Ogino T, Okuaki T et al (2007) Respiratory gated body diffusion weighted imaging avoiding prolongation of scan time: tracking only navigator echo (TRON) technique. Presented at the Joint Annual Meeting ISMRM-ESMRMB, Berlin, Germany, May 19-25, 2007

52. Schick F (2005) Whole-body MRI at high field: technical limits and clinical potential. Eur Radiol 15:946-959

53. Mürtz P, Krautmacher C, Träber F et al (2007) Diffusion-weighted whole-body MR imaging with background body signal suppression: a feasibility study at 3.0 Tesla. Eur Radiol 17:3031-3037

54. Willinek WA, Gieseke J, Kukuk G (2009) Parallel RF transmission in body MRI for reduced dielectric shading, improved B1 homogeneity and accelerated imaging at 3.0T: Initial clinical experience in 40 patients using MultiTransmit. Presented at the ISMRM 17th Scientific Meeting \& Exhibition, Honolulu, Hawai'i, USA, April 18-24, 2009
55. Deng J, Miller FH, Salem R et al (2006) Multishot diffusionweighted PROPELLER magnetic resonance imaging of the abdomen. Invest Radiol 41:769-775

56. Delfaut EM, Beltran J, Johnson G et al (1999) Fat suppression in MR imaging: techniques and pitfalls. Radiographics 19:373-382

57. Nagy Z, Weiskopf N (2008) Efficient fat suppression by sliceselection gradient reversal in twice-refocused diffusion encoding. Magn Reson Med 60:1256-1260

58. Takahara T, Zwanenburg J, Visser F et al (2009) Fat suppression with Slice-Selection Gradient Reversal (SSGR) revisited. Presented at the ISMRM 17th Scientific Meeting \& Exhibition, Honolulu, Hawai'i, USA, April 18-24, 2009

59. Weissleder R, Elizondo G, Wittenberg J et al (1990) Ultrasmall superparamagnetic iron oxide: characterization of a new class of contrast agents for MR imaging. Radiology 175:489-493

60. Weissleder R, Elizondo G, Wittenberg J et al (1990) Ultrasmall superparamagnetic iron oxide: an intravenous contrast agent for assessing lymph nodes with MR imaging. Radiology 175:494-498

61. Will O, Purkayastha S, Chan C et al (2006) Diagnostic precision of nanoparticle-enhanced MRI for lymph-node metastases: a meta-analysis. Lancet Oncol 7:52-60

62. Thoeny HC, Triantafyllou M, Birkhaeuser FD et al (2009) Combined ultrasmall superparamagnetic particles of iron oxide-enhanced and diffusion-weighted magnetic resonance imaging reliably detect pelvic lymph node metastases in normal-sized nodes of bladder and prostate cancer patients. Eur Urol 55:761-769

63. Senéterre E, Weissleder R, Jaramillo D et al (1991) Bone marrow: ultrasmall superparamagnetic iron oxide for MR imaging. Radiology 179:529-533

64. Von Schulthess GK, Schlemmer HP (2009) A look ahead: PET/MR versus PET/CT. Eur J Nucl Med Mol Imaging 36(Suppl 1):S3-9

65. De Jong PA, van Ufford HM, Baarslag HJ et al (2009) CT and 18 F-FDG-PET for noninvasive detection of splenic involvement in patients with malignant lymphoma. AJR 192:745-753

66. Pakos EE, Fotopoulos AD, Ioannidis JP (2005) 18F-FDG PET for evaluation of bone marrow infiltration in staging of lymphoma: a meta-analysis. J Nucl Med 46:958-963

67. Zijlstra JM, Lindauer-vander Werf G, Hoekstra OS et al (2006) 18F-fluoro-deoxyglucose positron emission tomography for posttreatment evaluation of malignant lymphoma: a systematic review. Haematologica 91:522-529

68. Terasawa T, Nihashi T, Hotta $T$ et al (2008) 18F-FDG PET for posttherapy assessment of Hodgkin's disease and aggressive Non-Hodgkin's lymphoma: a systematic review. J Nucl Med 49:13-21

69. Cook GJ, Fogelman I, Maisey MN (1996) Normal physiological and benign pathological variants of 18-fluoro-2-deoxyglucose positron-emission tomography scanning: potential for error in interpretation. Semin Nucl Med 26:308-314 